Comunicação e Sociedade, vol. 15, 2009, pp. 65-75

\title{
Un monde familier. Petite histoire du journal télévisé français
}

François Jost*

\section{Resumé}

Comment faire l'histoire du journal télévisé ? Ce texte part de l'idée que l'acte d'informer est double : celui-ci repose, d'une part, sur des assertions sérieuses quant à notre monde, d'autre part, sur un dispositif d'énonciation, qui permet de faire partager ces assertions à un public. Comment ses assertions sont-elles construites par le journal télévisé ? Que restitue-t-il de la réalité à laquelle il renvoie ? Et comment fait-il partager un environnement cognitif à son destinataire ? Voici donc quelques questions que nous traitons dans cet article.

Mots-clés: Télévision française, Journal télévisé, histoire.

Comment faire l'histoire du journal télévisé ? Et plus généralement, comment faire l'histoire de la télévision ? Ces questions d'ordre épistémologique sont rarement posées pour elles-mêmes. Pourtant toute histoire de la télévision ou du journal télévisé y répond implicitement par ses choix et son écriture. Deux grands types de récit dominent. Le premier est manichéen et découle d'une affirmation d'Umberto Eco, qui, j'en suis sûr, n'a pas souhaité une telle caricaturisation ; il oppose une paléo-télévision, verticale, fenêtre sur le monde, à une néo-télévision, ouverte à l'autre et à la convivialité et friande du mélange de genres (Eco, 1985). Le second, fondamentalement orienté par la téléologie, considère que l'histoire du journal télévisé est celle d'une libéralisation progressive de l'information par rapport au pouvoir politique.

L'histoire d'un programme comme le journal télévisé se complique encore du fait qu'il devient très souvent, dans l'esprit de ceux qui la racontent, indissociable de grands événements dont il a parlé : comment passer sous silence l'assassinat de Kennedy, qui a

\footnotetext{
* Professeur à la Sorbonne Nouvelle-Paris III, Directeur du Centre d'Etude des Images et des Sons Médiatiques. Francois. jost@univ-paris3.fr et francoisjost@ceisme.fr
} 
engendré l'une des premières éditions spéciales du journal télévisé de la première chaîne française, ou l'interdiction de tourner au Quartier Latin en 1968 ? Ce que l'on dit du journal télévisé est tellement lié à l'histoire tout court que, pour l'archiver, on s'est contenté pendant des années de conserver les reportages, les "sujets ", sans se soucier des présentateurs. Cette logique "professionnelle ", seulement attentive à la constitution d'archives dans lesquelles on pourrait piocher en fonction des besoins du présent (nécrologie d'un homme célèbre, rappel historique, etc.) explique aussi que l'histoire des formes soit souvent passée au second plan. Certes, il ne s'agit pas de tomber dans l'excès inverse et d'entreprendre une histoire, sans fin, de tous les changements de dispositif, déconnectée de la réalité, mais de trouver une alternative à une histoire du journal télévisé qui empile des dates sans cohérence d'ensemble, faisant tantôt référence à une décision politique, tantôt à une transformation institutionnelle, tantôt une rupture technique. Comment faire pour périodiser une histoire de l'information sans la calquer obligatoirement sur les tournants politiques (opération qui aboutit à des découpages comme " la télévision sous de Gaulle ", " la télévision sous Mitterrand ») ? Ce texte est, à sa manière, une réponse à cette question.

\section{Le spectacle du monde en voix over (1949-1954)}

Il est un point au moins sur lequel tout le monde s'accorde : le premier journal télévisé est diffusé le 29/6/49, à 21h. L'appellation journal télévisé est évidemment trompeuse. On parle alors des « actualités françaises ». Ce que les quelques centaines de téléspectateurs que compte la seule chaîne de télévision peuvent voir à l'époque est bien plus près des Actualités cinématographiques hebdomadaires projetées dans les salles de cinéma. Des reportages se succèdent, commentés par une voix over, simplement séparés par des cartons. Ces débuts donnent une résonance particulière à cette prédiction de Jean Thévenot en 1946, qui affirmait dans son livre L’Âge de la télévision et l'avenir de la radio : " La télévision s'adressera, avec les moyens de la radio, à un public qui attendra d'elle l'équivalent du cinéma » (p. 26). Encore faut-il rajouter qu'en l'occurrence, elle utilise les moyens du cinéma de deux façons : d'abord, par emprunt direct, en diffusant des images qui sont destinées d'abord aux salles obscures, ensuite par des moyens propres, puisqu'elle tourne ses propres images sur pellicule, en l'occurrence avec des caméras Bell Howell $16 \mathrm{~mm}$ sans enregistrement sonore. Les commentaires, en revanche, faits directement en cabine pendant que les reportages défilent, mobilisent bien en revanche les " moyens de la radio ». En sorte que se trouvent conjuguées deux temporalités : celle de l'image enregistrée, conçue pour un rythme hebdomadaire, qui identifie l'actualité à un bain dans lequel les spectateurs sont immergés pendant une semaine, et celui de la radio, qui est, au contraire, celle du direct, de l'instant, de l'événement qui se dissipe. C'est d'ailleurs de la radio que viennent les premiers journalistes de l'information télévisée.

Fondée sur le cinéma, la télévision sera longtemps, malgré elle, du côté du temps cinématographique, un temps contraint par la technique, par la durée des magasins, qui impose de prévoir l'événement (pour envoyer une équipe sur place), mais aussi d'anticiper sur son déroulement (comme lorsqu'il fallait deviner si l'attaque d'une équipe de 
football allait déboucher ou non sur un but: parfois on rend compte de matches de foot gagnés par 3-0 sans montrer le moindre but !).

Rencontre de deux médias, le cinéma et la radio, les actualités télévisées entretiennent aussi une parenté certaine avec les débuts du cinéma par l'articulation qu'elles font de deux médiums, l'image et la parole. Comment ne pas voir dans ce journaliste qui commente l'image au fur et à mesure dans la cabine de son studio, en ajoutant quelques plaisanteries, le cousin de ce bonimenteur du cinéma des premiers temps, qui improvisait à partir d'une trame un commentaire lié à ce qui se déroulait sur l'écran ? Comme celui-ci, le journaliste sélectionne l'information pertinente et prescrit au spectateur un parcours dans l'image, mais il y ajoute l'assurance que ces icônes sont aussi des indices ou, pour le dire plus simplement, que ces images renvoient à la réalité. Très vite, la musique va, comme dans le cinéma muet, orienter le sens, en fonction d'un catalogue de codes appropriés à des situations-types. Face à cette première période, nous sommes aussi démunis que face aux premiers films : même quand nous avons des reportages, ils sont muets, ou, pour mieux dire, mutilés, puisqu'il leur manque une partie essentielle des informations qui furent délivrées au spectateur par la voix. Une chose est sûre: malgré les similitudes, il existe une différence de taille entre le " conférencier » du cinéma des débuts et le journaliste des premiers journaux télévisés : tandis que ce premier s'appuyait sur des catalogues et des histoires connues par avance, le journaliste de 1949 n'avait parfois qu'à peine le temps de découvrir les images qu'il allait commenter. Le relevé des incidents lors de la diffusion nous apprend, par exemple, que, le $1^{\text {er }}$ juillet, "pendant la séquence de la 'mode d'été' et 'les acrobaties automobiles' le récepteur de la cabine était complètement déréglé tant et si bien que les reporters commentaient sans images ", incident qui se reproduit presque à l'identique le 6 juillet. Cette situation qui perdura plusieurs années, où le journaliste ne connaîtra le sens des images que par ce que lui en a dit le témoin direct de la réalité, le caméraman, place le journaliste " en cabine " dans une position qu'il partage avec son destinataire : il est certes le premier spectateur des images sur lesquelles il tient des informations, mais il reste spectateur, en sorte qu'il est dans une position assez similaire à celle de son destinataire. Position qui ne lui permet pas toujours d'observer confortablement la fameuse "fenêtre sur le monde ", dont on ne cesse de nous rebattre les oreilles. Parfois les vitres sont un peu opaques...

Et sur quel monde donne-t-elle cette prétendue fenêtre ? On imagine parfois que les rubriques du journal télévisé d'aujourd'hui, avec ces grandes séparations entre l'international et le national, la politique et le fait-divers, ont existé de tout temps. Il suffit de lire les conducteurs de ces premières « actualités" pour se convaincre du contraire. Rappelons d'abord dans quelles circonstances commencent les premières actualités télévisées : elles naissent pour rendre compte du Tour de France cycliste et s'arrêtent d'ailleurs avec son arrivée, trois semaines plus tard, pour ne reprendre que le 2 octobre, cette fois-ci de façon définitive. C'est dire que, dès l'origine, la liaison des informations télévisées et du spectacle est quasi-consubstantielle. Le détail des reportages le confirme. Voici, par exemple, ce que nous permet de dire l'examen des conducteurs de 4 journaux télévisés des premiers jours (du $1^{\text {er }}$ au 8 juillet 1949). La tranche d'information 
a alors une durée variable, qui oscille de 10 minutes (le $1^{\text {er }}$ juillet) à 17 minutes (le 17 juillet). Il y a de 5 à 8 reportages de durées tout à fait inégales (de 55" à 4'). Dans ce tout début, les reportages ont globalement d'autres sources que la télévision elle-même : sur les 27 reportages diffusés, 17 viennent directement du cinéma et, plus précisément, des actualités cinématographiques françaises, quatre viennent de la BBC, un du service cinématographique des armées, trois sont produits par l'équipe de la télévision, et cinq ont une origine inconnue. Cette origine cinématographique des images implique que, majoritairement, les images diffusées sont en $35 \mathrm{~mm}$ et non en 16 .

Le vendredi $1^{\text {er }}$ juillet s'enchaînent, dans l'ordre : "images du tour » (la première étape du tour), "images du sport »(la victoire du cycliste Gaston Reiff), "image du sport " (Grand prix de Longchamp), " image du sport » (Acrobaties aériennes), "images de Paris » (mode d'été), " images du monde » (appareil à nettoyer les plages), " images du monde » (Naufrage de la Reine Astrid, le 21 juin). Le monde est conçu comme un spectacle fournisseur d'images, où la politique n'a aucune place. Même chose ou presque le mercredi 6 juillet : "images du tour »(deux séquences), "images du monde » (images d'archive des manœuvres de la flotte occidentale), "image de Paris " (Les amis de l'homme), "images de France » (Versailles, Fête du 50 anniversaire de la chambre de commerce). Un an plus tard, le 12 avril 1950, l'équilibre est modifié, mais sans pour autant viser l'exhaustivité : "La vie politique » (congrès du parti communiste, 1'45"), " conflits sociaux " (la crise belge, 1'55”), Automobiles (course à Pau, 2'30”), Cyclisme (Paris-Camembert, 7’05) ... Dix minutes pour le sport, 3’40 pour la vie politique !

\section{Les mots ont un visage (1954-1964)}

Si le journaliste commentateur n'est, après tout qu'un " premier » téléspectateur, mieux informé que celui qui le regarde dans sa maison, il ne se "décolle " de son confrère cinématographique qu'au moment où apparaît sa propre image dans l'écran. Ce changement de formule date de 1954, mais il s'opère progressivement. Au début, jusqu'à trois journalistes interviennent en plateau (Pierre Tchernia, Claude Darget, Claude Joubert): ils lisent une série de nouvelles, puis commentent les reportages et font parfois l'interview d'un invité. En fait, le phénomène est progressif. Le 2 décembre 1954, par exemple, le journal télévisé se répartit de la façon suivante : 6 minutes de plateau et 15 minutes de reportages commentés : Météo (1'20”), Le prince Rainier à l'Élysée (1'05”), le Maréchal Juin reçoit le dragueur Garigliano (2'), Tempête en Grande-Bretagne (2'10”), Départ des 6 jours cyclistes de Bruxelles (1'15”), Pose de la première pierre de l'Héliport de Paris (0'55"), À Rome, campagne du silence (1'20") et à Paris opération moto silencieuse (4'55"). Comme on voit, les sujets ont encore des durées très diverses.

Si la réalité a pris un tour plus sérieux, moins spectaculaire que dans les tous premiers " journal télévisé », la vraie rupture est évidemment dans le lien nouveau que les actualités instaurent avec leur téléspectateur grâce au présentateur. Pour le fondateur de l'information télévisée, Pierre Sabbagh, cette invention aurait été une solution aux difficultés de fabrication des reportages, que la lourdeur du matériel empêchait de saisir les événements sur le vif. En fait, avec un peu de recul, il est clair que cette apparition 
coïncide surtout avec la prise de conscience très forte du directeur de la télévision de l'époque, Wladimir Porché, que la différence entre le cinéma et la télévision réside dans la relation d'intimité qu'elle peut tisser avec son spectateur. D'où cette recommandation qu'il adresse à tous ceux qui présentent une émission " de fixer la caméra puisqu'ils s'adressent non seulement à une salle, mais à des spectateurs isolés ${ }^{1}$. Du coup, le plateau prend progressivement de l'importance. Dans l'un des premiers journaux intégralement conservés, celui du 22 novembre 1956, il occupe la moitié du temps (onze minutes sur vingt-quatre). Néanmoins, il ne joue pas ce rôle de pivot que nous lui connaissons: le présentateur " lance » rarement les sujets, qui sont annoncés par un carton ou par une ponctuation visuelle, et il les "désannonce » encore moins. Soit il informe par la parole, soit assiste comme nous au spectacle du monde, dont la médiation devient elle-même un spectacle. L'espace du studio se revêt, en effet, de multiples signes de technologie: à côté du micro trônent deux téléphones, le présentateur a à sa disposition un bouton bien visible qui lui permet d'envoyer les reportages. Premier spectateur, il est aussi à présent premier auditeur: pendant de longues minutes, il va écouter, comme nous, la voix du correspondant à Moscou s'échappant d'un haut-parleur posé sur son bureau. Après une allocution du président du conseil sur la pénurie d'essence, deux reportages s'enchaînent sans aucun lancement de la part du présentateur. Seul un carton introduit le premier ( Rokossovski s'en va »). De façon générale, les images qui sont montrées proviennent encore de la presse filmée, sauf, cas exceptionnel, quand un envoyé spécial a été dépêché sur place. Les reportages filmés par la télévision française concernent l'espace proche (plutôt parisien, donc). La musique accompagne systématiquement l'image qui, sans le commentaire, serait muette et "meuble " parfois 10 à 15" de silence. Parfois, un son d'ambiance a été ajouté, comme celui d'un moteur d'avion, dont l'intensité constante cadre mal avec les changements d'échelle de plans ou les changements de points de vue. Dans ce contexte, les «nouvelles de dernière minute " sont données par la seule parole à la fin du journal par un autre journaliste.

Dans les années qui suivent, trois éléments vont renforcer le lien avec le téléspectateur.

Le premier est la régularité du rendez-vous : en 1957, la diffusion du journal télévisé passe de $20 \mathrm{~h} 15$ à 20h, heure qui ne bougera plus jusqu'à aujourd'hui, à quelques exceptions ponctuelles. L'information se rapproche de l'heure du repas (en France, on dîne entre 19 et 20 h), qui réunit l'ensemble de la famille.

Le deuxième est la personnalisation du présentateur: à partir de 1960, cinq journalistes sont choisis pour présenter le journal télévisé à tour de rôle: Claude Darget, Léon Zitrone, Raymond Marcillac, Michel Droit et Joseph Pasteur².

Le troisième est le renforcement de l'intimité par l'effacement de la médiation entre le présentateur et le téléspectateur. Si l'injonction de regarder celui-ci dans les yeux date des années 50, ce n'est qu'en 1963 qu'est mis en place une innovation technique qui permet de simuler une situation de conversation franche et directe par l'entremise du regard-caméra: le prompteur.

\footnotetext{
${ }^{1}$ Mon programme $\mathrm{n}^{\circ} 78,17 / 4 / 1954$.

${ }^{2}$ En 60 , le journal télévisé a quatre éditions: $13 \mathrm{~h}$, info politiques et faits divers ; 19h15, enquêtes, reportages et magazines ; 20h15: politique intérieure et ext. 22h30, résumé de toute l'actualité en 30 minutes.
} 


\section{3. « Laisser parler les images » (1963-1968)}

C'est pour aller contre cette place primordiale du présentateur qu'Alain Peyrefitte, ministre de l'information, vient présenter lui-même la nouvelle formule dans le cadre du journal télévisé en avril 1963. C'est en tout cas comme cela qu'il présente les choses: il faut, dit-il, "supprimer les commentaires pour laisser parler les images ou les faits ou alors des dialogues ", ce qui, selon lui, " marquera un progrès vers l'objectivité et la dépolitisation ». Et il déclare aussi à l'AFP: «Le génie de la télévision, c’est l'image... Ce qui compte, ce qu'il faut développer de plus en plus, ce sont les films d'actualité, reportages, photographies, dessins, etc. Remplacer les propos par l'image photographique de l'objet, c'est-à-dire l'événement [...] Le présentateur va être transformé en meneur de jeu qui devra s'effacer devant les images ou le témoin qui parlera de ce qu'il a vu lui-même ou devant les spécialistes d'une question particulière ». La mise en scène du journal télévisé illustre parfaitement cette conception: derrière le présentateur vont venir s’inscrire sur un grand écran des incrustations qui permettront aussi la vision d'une relation avec le monde. C'est le culte du témoignage oculaire prôné par le magazine Cinq colonnes à la une, et, ce faisant, pour le ministre, le vrai énonciateur de l'information, c'est la réalité elle-même, l'image photographique devenant l'événement. L'ère de « l'image qui parle toute seule " s'ouvre. L'adoption de la caméra Eclair-Coutant, beaucoup plus légère et moins sonore que les précédentes, va faciliter la prise de vue à l'épaule et l'arrivée du magnétoscope va permettre la réutilisation des images. Pourtant, c'est tout le contraire de l'objectivité qui se met en place; le journal télévisé est piloté directement par le ministre de l'information et certaines informations censurées par le rédacteur en chef.

Tout le pouvoir étant donné à l'image, le présentateur devient donc un meneur de jeu chargé de la circulation de la parole, médiateur entre les images-événements et les "spécialistes d'une question particulière ". Du même coup, l'information ne se limite plus au visible, elle connaît une extension du côté de l'intelligible, certains experts étant réputés éclairer la réalité par des mots, des schémas ou des dessins, des graphiques ou des maquettes. On entre dans ce que l'on a appelé le "journalisme d'examen ", qui se donne pour tâche de "produire de l'invisible» (Brusini et James, 1982). La même année se met d'ailleurs en place une véritable " rédaction » avec des services spécialisés, "calqués sur l'organisation des journaux de presse écrite : politique, économie, social, étranger, faits divers, société, sport, culture » (Asline 1990 : 70)

En 1964 est lancée une seconde chaîne, qui mettra plusieurs années à s'imposer (un sondage de 1966 révèle que $70 \%$ de ceux qui peuvent la recevoir ne l'ont jamais regardée ${ }^{3}$ ). Ce n'est qu'en 1970 qu'on séparera la programmation des deux chaînes. Au début, la seconde chaîne n'a un journal télévisé qu'à 22 heures et elle "n'ouvre " qu'à 19h ou 19h30 jusqu'en 1972. L'année 1968 va être une année-charnière à bien des égards: d'abord, parce que le lien avec le pouvoir prend la forme d'une censure sévère pendant le mois de Mai, ce qui aboutit à un mouvement de grève très dur des journalistes, qui, passés les événements, sont massivement mis à la porte. Ensuite, parce que, du point de vue télévisuel, intervient une rupture dont la coïncidence temporelle est surprenante: en cette année où les étudiants

${ }^{3}$ Source : Jacques Mousseau, Christian Brochand, Histoire de la télévision, Nathan, Paris 1982. 
ont stipendié la "société de consommation ", la publicité de marque fait son apparition, ce qui va entraîner très vite la rigidification des grilles, avec ses carrefours dictés par les «écrans publicitaires".

\section{Le vedettariat comme stratégie de concurrence (1969-1986)}

1969. Après le tourbillon de l'année précédente, s'ouvre une nouvelle période, celle de la concurrence des deux chaînes. La rédaction est séparée en deux: Information Première, sous la direction de Pierre Desgraupes, et 24 beures sur la 2, dirigé par Jacqueline Baudrier. Déjà en 1968, la programmation des deux journaux avait été décalée : 20h sur TF1 et 19 h30 sur A2. En 1969, celui de TF1 passe à 19h45 et celui de la seconde chaîne à $20 \mathrm{~h} 30$, puis à $19 \mathrm{~h} 30$. Non seulement, ce journal commence plus tôt, mais il innove: d'une part, il est en couleurs, d'autre part, il dure une heure, comprenant deux parties, l'une de magazine, l'autre d'informations. Bien que les deux rédactions cohabitent dans le même lieu, elles cultivent le secret pour lutter contre leur concurrente. Les journalistes les plus turbulents ont été chassés de la première chaîne et déplacés sur la deuxième. Les deux rédactions ne vont pas tarder à s'opposer sur leur philosophie quant aux présentateurs: la première chaîne va jusqu'au bout de la logique du vedettariat, dont les prémices sont observables dans les années 60 , en mettant à la tête du journal un journaliste qui présentera tous les 20 heures de la semaine, sur le modèle de W. Cronkite aux USA. Joseph Pasteur, qui est censé lui ressembler, est choisi.

En 1973, la troisième chaîne voit le jour. Pendant les deux premières années, le journal télévisé clôture les programmes et un 20h est lancé en 1975. La concurrence va prendre un nouveau tournant avec l'éclatement de l'ORTF, l'Office de Radio-Télévision française, qui jusqu'alors administrait toutes les chaînes de télévision et de radio. Les chaînes de télévision deviennent trois sociétés autonomes, TF1 (Télévision Française 1), Antenne 2 et France Régions 3. Bien qu'elles soient toutes les trois publiques, leur concurrence s'accentue, du fait que la redevance leur est distribuée notamment en fonction de leur audience et que leur quota de publicité diffusable est limité à $25 \%$ de leur budget. Le vedettariat devient alors une stratégie annoncée de différenciation. Il ne s'agit plus simplement de créer familiarité et intimité avec le téléspectateur, il s'agit d'utiliser le présentateur comme un des traits de la chaîne conçue comme une marque. "Personnifier le journal pour fidéliser le public », devient le mot d'ordre du rédacteur en chef de l'information de TF1, Christian Bernadac (cité dans Asline 1990: 98). Roger Gicquel est choisi pour le 20h, Yves Mourousi pour le $13 \mathrm{~h}$. A2 en vient à la même politique et met à l'antenne, un journaliste qui occupera trente ans cette fonction, d'abord sur Antenne 2 ensuite sur TF1, Patrick Poivre d'Arvor. Le présentateur n'est plus seulement une parole ou la "voix de la France », comme le dit le Président de la République de l'époque, Georges Pompidou, il est un corps. Bien qu'il soit souvent présenté comme un homme-tronc, ses moues, ses mimiques accompagnent l'information et lui donnent du sens. Loin de rester impassible devant le spectacle du monde, comme un énonciateur neutre, qui se conterait de nous délivrer de l'information, Roger Gicquel parle beaucoup (près de la moitié du temps du journal), il semble souffrir devant certaines nouvel- 
les: il n'est pas seulement ce locuteur L, responsable de l'énonciation, mais un être du monde, en bref, un être humain ${ }^{4}$. Le journal télévisé n'est pas seulement personnifié, il est humanisé.

En 1978 survient un changement technique, qui va apporter de profondes modifications à la représentation du monde: le remplacement du support cinéma par la vidéo. L'allongement du temps possible d'enregistrement, la suppression du développement de la pellicule raccourcissent l'intervalle entre l'événement et la diffusion d'un reportage. C'est l'un des éléments qui vont permettre la mise en place d'une édition de 13 heures sur TF1. Dès lors, les journaux de la mi-journée et du soir se différencient: "À 13 heures, l'information est en mouvement, il faut la saisir au vol [...]. À 20 heures, la journée est terminée, l'actualité est bouclée, on raconte ce qui s'est passé » (Pierre Géraud, cité par Asline, 1990 : 99). De ce constat, l'équipe du présentateur-vedette de l'époque, Yves Mourousi, tire le dispositif du 13 heures: comme à ce moment de la journée, le temps n'est pas venu pour " raconter ce qui s'est passé ", le journal télévisé reçoit beaucoup d'invités et déplace son plateau du studio au monde et s'organise autour de grands directs: depuis la Place Rouge de Moscou ou la place Tian'anmen, à l'Institut Pasteur, à l'Académie française ou sur un porte-avion...

L'arrivée de la gauche au pouvoir en 1981 provoque des remous dans les rédactions. TF1 s'interroge sur le vedettariat et préfère une formule avec trois présentateurs qui tournent. La chaîne essaye aussi une présentation en duo, tandis qu'Antenne 2 confie la présentation du journal télévisé à une femme, Christine Ockrent, en alternance avec le présentateur en place depuis 7 ans, Poivre d'Arvor. En 1982 est mise en place la Haute Autorité, qui a pour mission de réguler l'audiovisuel, mettant fin à la subordination directe des chaînes aux gouvernements. Autre conséquence du changement idéologique dont la critique du vedettariat est le symptôme, la suppression d'émissions à succès jugées dégradantes. Tous ces faits amènent le journal télévisé de la deuxième chaîne à prendre l'avantage en termes d'audience. Le studio, d'abord lieu de médiatisation est entre-temps devenu le lieu d'où part l'événement, comme le montre très clairement l'évolution des génériques. En 1965, le journal s'ouvrait sur l'image d'un reporter qui rapportait des images avec sa caméra de cinéma. En 1984, le générique du journal télévisé de TF1 est un écran de radar au centre duquel se trouve un 1, et dont le balayage est effectué par un rayon formé par une ligne de logos TF1 imbriqués. Du centre surgissent les images illustrant les titres. Sur Antenne 2, l'image de la présentatrice arrive du fond de l'écran sur un décor formé par le planisphère et disparaît de la même façon à la fin du journal, le sourire aux lèvres, avec la satisfaction d'avoir clarifié le désordre du monde. L'infographie prend une place importante dans la présentation du journal affirmant la primauté de la puissance technologique dans la construction de l'information. Au plateau est dévolu un rôle de scansion et de clarification : les reportages sont annoncés par des bandeaux rouges sur Antenne 2, les rubriques sont nommées et symbolisées

\footnotetext{
${ }^{4}$ Dans Le Dire et le dit, Oswald Ducrot distingue entre le sujet parlant, qui est l'instance empirique de la parole, et le locuteur $L$, qui est le sujet responsable de l'énonciation en tant que telle et le locuteur - l'être du monde, qui a, en autres, la propriété de parler, mais aussi de ressentir.
} 
par des images inscrites dans un petit rectangle à gauche ou à droite du présentateur. L'infographie prend une place ascendante pour expliquer les phénomènes difficiles à rendre visible, comme l'économie.

En 1984, les chaînes publiques commencent à émettre toute la journée, sans doute sous la pression de Canal+ qui vient d'être lancé, ce qui entraîne la création de nouveaux journaux télévisés le matin.

\section{Le règne du vécu (depuis 1987)}

1987: TF1 est privatisée et son journal repasse rapidement en tête des audiences. De $36 \%$ de part d'audience contre $45 \%$ pour Antenne 2, en 1985 , TF1 atteint $46 \%$ en 1988 , contre $22 \%$ à sa concurrente. Des multiples conséquences de la privatisation sur les contenus, j'en retiendrai deux qui vont toucher durablement la chaîne et qui, bien sûr, ont la même cause : la soumission de la chaîne aux impératifs du marché et aux recommandations du marketing. Le journal télévisé de TF1 va adapter son contenu au public disponible. Le 22 février 88 débute un 13 heures présenté par Jean-Pierre Pernaut, fabriqué en partenariat avec la presse quotidienne régionale, qui va mettre l'accent sur les régions, la tradition, les contribuables, valeurs qui sont celles du public présent devant le petit écran dans cette tranche horaire: les provinciaux, qui ont le temps de rentrer déjeuner chez eux, les artisans, les commerçants, les personnes âgées. La directrice de l'information de l'époque, Michèle Cotta, exprime sa conviction que "la télévision, ce n'est plus les gens venus d'en haut» (cité par Roberts, Garrigos 2006 : 77). Tous les sujets du journal télévisé vont dans ce sens: il s'agit de montrer les difficultés que rencontrent les "petits" face aux administrations en tout genre. Quelques années plus tard, les producteurs des premiers reality shows lancés par TF1 useront exactement des mêmes mots et justifieront leurs programmes par les mêmes arguments. Dès lors, d'un bout à l'autre de la grille, le mot d'ordre sera " proximité ». Après avoir créé et resserré le lien avec le téléspectateur par la personnalité du présentateur, le journal télévisé se donne une nouvelle stratégie: il construit des reportages dans lesquels le témoignage vécu reçoit la charge de la preuve. "L'homme est mesure de toute chose ", comme le prétendait le sophiste Protagoras. D'une étude que je mène alors pour A2 et FR $3^{5}$, il ressort que l'angle des deux grandes chaînes nationales sur la réalité diffère. Sur TF1, la dimension "humaine " des acteurs de la réalité est mise en exergue, qu'il s'agisse d'un syndicaliste en grève ou d'un ministre ou, même du Président de la République, dont on dit, le jour où il visite une exposition consacrée aux Vikings: "Mitterrand, qui a dû essayer d'oublier ses soucis » (31/3/1992). Rien de plus parlant, à cet égard, que de comparer le traitement d'un événement historique le même jour sur deux chaînes. Nous sommes le 31 octobre 91 et les négociations de Madrid entre Israéliens et Palestiniens viennent de s'ouvrir. Pour commencer, TF1 comme A2 montrent des diplomates des deux pays en train de discuter dans les couloirs pour se prendre à partie quelques minutes plus tard. La suite, en revanche, sépare les

${ }^{5}$ «Etude pour l'optimisation des journal télévisé du début de soirée », SORGEM, 28 avril 1992. 
deux éditions. Pour TF1, il s'agit de montrer avec le sujet suivant que les discussions de Madrid ont des " conséquences très directes sur la vie des Arabes et des Israéliens »: le reporter a confectionné un " portrait de deux familles, l'une juive, l'autre arabe » ayant perdu toute deux un enfant dans la guerre et qui souhaitent la paix. Et le reportage de conclure, sur le plan d'une route sur laquelle marchent des soldats: "loin de Madrid, voici un aperçu de la route qu'il faut encore parcourir vers la paix ». Le lieu de la négociation semble bien loin de la réalité du terrain, seule authentique. Sur Antenne 2, se succèdent un direct à Madrid avec l'envoyé spécial de la chaîne, un portrait du chef de la délégation palestinienne, un portrait de G. Habache, dissident de l'OLP. L'événement est vu au travers des négociateurs, des "grands ", comme aurait dit la directrice de l'information de TF1, et pas des « petits » qui vivent la guerre au quotidien. Du témoin oculaire médiateur de la réalité, du reporter sur le terrain dont le journal télévisé nous diffuse la vision dans les années 60 , nous sommes passés au témoignage des « acteurs » de la réalité. Il s'agit moins de montrer, de rendre visible ce qui est lointain que de faire connaître par le biais du récit de vie. Le vécu a pris le pas sur le $v u$. Ce retournement copernicien de l'information, qui tourne autour de la sensation subjective des " gens " plutôt que du regard que porte sur eux le journaliste, a partie liée, évidemment, avec l'arrivée des reality shows sur la chaîne. Le témoignage et le récit de vie, le " portrait » comme mode de connaissance de la réalité envahissent la grille, qu'il s'agisse de suppléer les manques de la police pour retrouver un enfant disparu (Perdu de vue), de réconcilier un couple (L'Amour en danger) ou de réparer les erreurs de la justice (Témoin $n^{\circ} 1$ ). Plutôt que de s'opposer à cette approche, A2 fera tout pour la copier, sans volonté de se séparer des méthodes de la chaîne privée.

Depuis les années 90, les journaux télévisés n'ont pas connu de changements majeurs. De 1995 à 2006, le plateau de TF1 ne connaîtra aucune modification et celui de France 2 se stabilisera entre la fin des années 90 et 2006. Les présentateurs mis en place par TF1 en 1988 ont fini par faire partie du paysage. Bien sûr, l'analyse de détail révèle des différences importantes, surtout en ce qui concerne le 13 heures. La chaîne publique n'a pas osé s'adapter aussi cyniquement que la chaîne privée au public disponible et, même si elle s'est teintée progressivement d'un ton plus régional, elle évite encore de relayer l'idéologie populiste de TF1. Il est toutefois un point sur lequel les deux chaînes s'accordent : le lien quasi auctorial qui unit le présentateur à son journal. Lors du lancement parallèle de leur nouveau plateau à la rentrée 2006, les deux chaînes recoururent au même procédé de la signature du journal, dès le générique, par le présentateur (13 heures Jean-Pierre Pernaut, pour TF1, ou 20 heures, David Pujadas, pour France 2). En même temps, l'une et l'autre accordent une place accrue à l'effet-fenêtre sur le monde, tout en gardant leur différence d'angle : tandis que France 2 suggère, à 20h, les lumières d'une ville abstraite, TF1 donne sur le tissu urbain qui l'entoure dans la réalité, comme si le présentateur se trouvait au centre du monde, si proche pourtant de la cité qui vit juste derrière le studio. Ainsi se trouvent réconcilier deux exigences paradoxales: la personnalisation de l'information et la promesse d'une fenêtre ouverte sur un paysage objectivement saisi par les caméras. En juin 2008, le présentateur qui présentait depuis 20 ans le 20 heures de TF1, Poivre d'Arvor, connut les limites de cette appropriation du 
journal télévisé par son présentateur: il fut « remercié » du jour au lendemain et remplacé par une femme de 20 ans sa cadette.

Une histoire aussi rapide de près de 60 ans d'histoire du journal télévisé est évidemment lacunaire. Du double point de vue qui est le mien, je la résumerai encore plus cavalièrement en soulignant, pour conclure, deux points qui semblent importants. Le premier est la modification du modèle de la vérité qui s'est opéré. Si dans les années 60, celle-ci venait du visible, du témoignage oculaire des envoyés spéciaux ou des reporters, elle se fonde aujourd'hui sur un tout autre modèle: le vécu a autorité, il authentifie une information. Le second est évidemment la starification du journaliste, qui, on l'a vu, est un phénomène complexe, qui engage aussi bien un lien d'intimité et de familiarité avec le téléspectateur qu'un besoin de placer le studio, puis la chaîne, au centre de l'information, pour ne pas dire de l'événement. Ce recentrement sur la chaîne imposerait de poursuivre cette compréhension des transformations du journal télévisé en fonction de la grille et, plus spécialement, des émissions "locomotives ", du lead in, comme disent les Américains, qui renforcent l'allégeance et la fidélité du téléspectateur à une chaîne.

\section{Bibliographie}

Asline, Jacques (1990), La Bataille du 20 heures, Paris, Acropole.

Brusini, Hervé et James, Francis (1982), Voir la vérité, Paris, PUF.

Ducrot, Oswald (1984), Le Dire et le dit, Paris, Minuit.

Eco, Umberto (1985), "TV : la transparence perdue », La Guerre du faux, Paris, Livres de poche n. ${ }^{\circ} 064$. Sperber, D. et Wilson, D. (1989) La Pertinence, trad. Fr., Minuit.

Thévenot, Jean (1946), L’Âge de la télévision et l'avenir de la radio, Paris, Éditions ouvrières. 